\title{
Use of botulinum toxin in stroke patients with severe upper limb spasticity
}

\author{
B B Bhakta, J A Cozens, J M Bamford, M A Chamberlain
}

\begin{abstract}
Objectives-Spasticity can contribute to poor recovery of upper limb function after stroke. This is a preliminary evaluation of the impact of botulinum toxin treatment on disability caused by upper limb spasticity after stroke.

Methods-Seventeen patients with severe spasticity and a non-functioning arm were treated with intramuscular botulinum $A$ neurotoxin (median age at treatment 54.5 years; median time between onset of stroke and treatment 1.5 years). Baseline and assessments two weeks after treatment were compared to assess efficacy. The duration of improvement in disability was documented. Outcome measures used were: passive range of movement at the shoulder, elbow, wrist, and fingers; modified Ashworth scale to assess spasticity of biceps and forearm finger flexors; an eight point scale to assess the degree of difficulty experienced by the patient or carer for each functional problem defined before treatment; the presence of upper limb pain. The biceps, forearm finger flexors, and flexor carpi ulnaris were treated with intramuscular botulinum toxin. Up to a total dose of 400-1000 mouse units (MU) of Dysport (Speywood) or 100-200 MU of BOTOX ${ }^{\circledR}$ (Allergan) was used in each patient.

Results-Functional problems reported by the patients before treatment were difficulty with cleaning the palm, cutting fingernails, putting the arm through a sleeve, standing and walking balance, putting on gloves, and rolling over in bed. Hand hygiene improved in 14 of 17 patients; difficulty with sleeves improved in four of 16; standing and walking balance improved in one of four; shoulder pain improved in six of nine; wrist pain improved in five of six. Passive range of movement at shoulder, elbow, and wrist improved after treatment. Benefit was noted within two weeks and lasted one to 11 months. No adverse effects occurred.

Conclusion-This preliminary study suggests that intramuscular botulinum toxin is a safe and effective treatment for reducing disability in patients with severe upper limb spasticity.
\end{abstract}

$(\mathcal{A}$ Neurol Neurosurg Psychiatry 1996;61:30-35)

Keywords: botulinum toxin; spasticity; stroke
Despite considerable rehabilitation effort, the prognosis for recovery of upper limb function after stroke remains poor. Of patients with an initially paralysed arm, only $4 \%$ to $5 \%$ regain normal function and up to $28 \%$ experience no recovery. ${ }^{1}$ Because upper limb function is essential for many tasks of daily living, impairment contributes to reduced quality of life and increases dependence. Even in patients with complete arm paralysis, spasticity can be an important contributor to disability, by causing pain or interfering with hygiene and dressing.

The mainstays of conventional treatment for severe upper limb spasticity include physiotherapy and systemic medication (baclofen and dantrolene). Despite these treatments, many patients remain disabled due to the spasticity. Moreover, the amount of systemic antispastic medication required may cause adverse drug reactions such as drowsiness. In addition, unwanted generalised muscle weakness may further increase disability-for example, reduction in ability to transfer or stand because of loss of tone in the hip and knee extensor muscles. To avoid these systemic effects local treatment with phenolic nerve and motor point blocks have been used in patients with severe upper limb spasticity. ${ }^{2}$ Although this treatment can be effective, it may cause dysaesthesia and local tissue necrosis in the treated arm. This treatment is currently not recommended for upper limb spasticity when sensation is preserved.

Intramuscular botulinum toxin $A$ offers the possibility of local treatment of spasticity without affecting sensation. It is an established treatment for squint, ${ }^{3}$ blepharospasm, ${ }^{4}$ hemifacial spasm, ${ }^{5}$ torticollis, ${ }^{6}$ and focal dystonias. ${ }^{7}$ More recently, it has been used to treat limb spasticity after stroke, traumatic brain injury, and multiple sclerosis. ${ }^{89}$ Botulinum toxin irreversibly blocks the release of acetylcholine from the nerve endings at the neuromuscular junction. Unlike alcohol and phenol nerve blocks, it has a selective action on motor nerves without affecting sensory nerve conduction. Preserved perception is an integral part of maximising motor recovery after stroke and therefore treatments that do not cause sensory disturbance have a theoretical advantage over non-specific local treatments.

The aims of this open pilot study were: (1) to identify the disabilities experienced by patients as a consequence of severe upper limb spasticity after stroke, (2) to assess the impact of treatment with botulinum toxin on impairments and disabilities using a new patient 
defined assessment, (3) to establish the safety of treatment in this group of patients.

\section{Materials and methods}

After stroke the resultant upper limb impairments and disabilities vary considerably. Therefore, to make a meaningful assessment of the impact of botulinum toxin, we treated a relatively homogeneous population. Patients were recruited according to the following criteria: (1) stroke was the cause of the impairments, (2) severe upper limb spasticity was affecting self care and had not been improved by conventional treatment such as physiotherapy, systemic antispastic medication, and use of orthoses, (3) there was absence of useful upper limb movement, (4) no previous local antispastic treatment had been used, and (5) informed consent was obtained.

COHORT

Seventeen patients (eight men, nine women) were recruited. Nine patients had right hemiplegia. None of the patients had significant language problems which affected comprehension of the outcome assessments used in this study. Median age at onset of stroke was 54.5 (range 16.3-68.5) years. Median time between onset of stroke and treatment with botulinum toxin was 1.5 (range $0.3-42.5$ ) years. Fourteen patients had complete upper limb paralysis. The remaining three had slight voluntary muscle activity but the resulting movement was not functionally useful. In these patients, the upper limb assumed a flexed posture at rest as a result of severe spasticity.

\section{ASSESSMENTS}

Before treatment and two weeks after treatment assessments of impairment and disability were made. Patients were followed up until they reported loss of initial functional benefit. Some patients were having physiotherapy for other disability problems such as mobility and seating. Treatment for these concurrent problems remained unaltered during this study.

TREATMENT

The treatment consisted of a single course of intramuscular botulinum toxin to the biceps brachii, flexor digitorum profundus, flexor digitorum superficialis, and flexor carpi ulnaris. The rationale for injecting these muscles is based on the clinical observation of the resting position of the upper limb in these patients. Spasticity in the biceps muscle caused the elbow to assume a flexed position in 15 of 17 patients. In all patients there was flexion at the proximal interphalangeal and metacarpophalangeal joints as well as flexion of the wrist related to spasticity of the flexor digitorum superficialis. Flexor digitorum profundus spasticity accounted for the flexion at the distal interphalangeal joints in 16 of 17 patients. In six of 17 patients the resting wrist position was not only flexed but also ulnar deviated suggesting that the flexor carpi ulnaris was also involved. These muscles were localised by standard anatomical landmarks as used in needle EMG. Standard dilutions of botulinum toxin in normal saline as recommended in the drug information leaflets were used. During the course of this study the preparation of botulinum toxin available to us changed. Initially Dysport (Speywood) and subsequently BOTOX ${ }^{\mathbb{R}}$ (Allergan) was used. The potency of both preparations is measured using a bioassay: one mouse unit (MU) is the median lethal intraperitoneal dose for one mouse. The potency of the BOTOX ${ }^{k}$ mouse unit and the Dysport mouse unit differ. Although there is no established ratio of potencies it is believed that I MU of BOTOX ${ }^{\mathrm{k}}$ is equivalent to about 4-5 MU of Dysport. ${ }^{10}$ In the present study, this equivalence ratio was used to maintain similar doses of botulinum toxin received by the patients. Dysport was used at a dilution of $500 \mathrm{MU}$ in $2.5 \mathrm{ml}$ of normal saline. BOTOX ${ }^{\circledR}$ was used at a dilution of $100 \mathrm{MU}$ in $2.5 \mathrm{ml}$. Table 1 shows the doses of botulinum toxin given to each muscle in each patient. Although the Ashworth scale was used to judge spasticity before treatment, there was a ceiling effect in some patients. This was particularly evident when rating spasticity in the forearm finger flexors, as all the patients fell into the highest category. Therefore in addition to the Ashworth grading, the dose given to individual muscles was based on clinical impression of spasticity, which was judged

Table 1 Doses of botulinum toxin used in each muscle

\begin{tabular}{|c|c|c|c|c|c|c|c|c|c|}
\hline \multirow[b]{2}{*}{ Patient } & \multirow{2}{*}{$\begin{array}{l}\text { Age at onset } \\
\text { of stroke }(y)\end{array}$} & \multirow{2}{*}{$\begin{array}{l}\text { Time between } \\
\text { stroke and } \\
\text { treatment }(y)\end{array}$} & \multirow[b]{2}{*}{ Drug } & \multicolumn{5}{|c|}{ Dose of botulinum toxin used (MU) } & \multirow{2}{*}{$\begin{array}{l}\text { Functional } \\
\text { benefit after } \\
\text { treatment }\end{array}$} \\
\hline & & & & Biceps & $F D P$ & $F D S$ & $F C U$ & Total dose & \\
\hline 1 & $53 \cdot 5$ & 0.4 & Dysport & 400 & 300 & 300 & 0 & 1000 & Absent \\
\hline 2 & $61 \cdot 1$ & $1 \cdot 4$ & Dysport & 200 & 100 & 100 & 100 & 500 & Present \\
\hline 3 & $47 \cdot 4$ & $1 \cdot 1$ & Dysport & 200 & 200 & 100 & 0 & 500 & Present \\
\hline 4 & $59 \cdot 8$ & $1 \cdot 0$ & Dysport & 200 & 150 & 100 & 0 & 450 & Present \\
\hline 5 & $42 \cdot 0$ & $5 \cdot 0$ & Dysport & 200 & 100 & 100 & 100 & 500 & Present \\
\hline 6 & $54 \cdot 5$ & $1 \cdot 0$ & Dysport & 200 & 100 & 100 & 100 & 500 & Present \\
\hline 7 & $31 \cdot 5$ & $11 \cdot 2$ & Dysport & 400 & 100 & 150 & 100 & 750 & Present \\
\hline 8 & $42 \cdot 0$ & $3 \cdot 8$ & Dysport & 100 & 150 & 150 & 0 & 400 & Present \\
\hline 9 & $47 \cdot 6$ & 1.5 & Dysport & 100 & 125 & 125 & 125 & 475 & Present \\
\hline 10 & $58 \cdot 4$ & 0.9 & Dysport & 200 & 100 & 100 & 100 & 500 & Present \\
\hline 11 & $43 \cdot 1$ & 0.6 & Dysport & 0 & 150 & 150 & 200 & 500 & Present \\
\hline 12 & $16 \cdot 3$ & $42 \cdot 6$ & BOTOX ${ }^{\circ}$ & 80 & 50 & 50 & 0 & 180 & Present \\
\hline 13 & $68 \cdot 5$ & $10 \cdot 0$ & BOTOX $®$ & 100 & 50 & 50 & 0 & 200 & Present \\
\hline 14 & $59 \cdot 0$ & $4 \cdot 0$ & ВОТОХ $®$ & 0 & 50 & 50 & 0 & 100 & Present \\
\hline 15 & $55 \cdot 0$ & $3 \cdot 5$ & ВОTOX $®$ & 50 & 50 & 50 & 0 & 150 & Absent \\
\hline 16 & $58 \cdot 8$ & $2 \cdot 0$ & ВОTOX $®$ & 70 & 30 & 50 & 0 & 150 & Absent \\
\hline 17 & $60 \cdot 9$ & 0.3 & BOTOX $₫$ & 50 & 30 & 50 & 0 & 130 & Present \\
\hline
\end{tabular}

FDP = flexor digitorum profundus; FDS - flexor digitorum superficialis; FCU = flexor carpi ulnaris 
by the passive resistance to movement of the wrist, fingers, and elbow. The total dose per patient did not exceed $1000 \mathrm{MU}$ of Dysport or $200 \mathrm{MU}^{\mathrm{N}}$ of BOTOX ${ }^{\circledR}$.

\section{MEASURING IMPAIRMENT}

The modified Ashworth grading ${ }^{11}$ was used to clinically assess spasticity of the biceps brachii and the forearm finger flexors.

Range of passive joint movement at the shoulder, elbow, and wrist was measured by protractor goniometry. There is no scale that allows easy recording of the finger position in these patients. A five point scale was therefore devised to record finger position at rest and at maximal passive finger extension. The scaling was determined by the number of the assessor's fingers that could be easily inserted into the subject's palm. The patient's fingers were deemed to be fully extended when four fingers could be placed on to the palm of the hand without difficulty and fully flexed when even one finger could not be easily inserted into the palm (no fingers - fully closed; one finger-1/4 open; two fingers-1/2 open; three fingers3/4 open; four fingers-fully open). The degree of finger flexion was rated with the wrist in its resting position of palmar flexion.

Initially we attempted to use a visual analogue scale to determine the severity of pain but this was later abandoned as these patients had difficulty in completing the scale. Subsequently the presence and location of pain were recorded at rest and on passive movement of the arm before and after treatment. Any change in pain reported by the patient was documented.

\section{MEASURING DISABILITY}

Our preliminary experience found the Barthel index $^{12}$ to be insensitive to change in disability reported by patients. Therefore a patient defined goals assessment was designed to measure individual changes at the disability level, using specific self care problems selected by patients. An item bank of self care activities had been developed by an open interview with the patients. The patient or the carer described the areas of difficulty with self care that they considered were related to spasticity in the arm. For each of these identified problems, the patient rated the degree of difficulty on a four point scale ( $1=$ no difficulty; $2=a$ little difficulty; $3=$ moderate difficulty; $4=a$ great deal of difficulty) before and after treatment. The carer completed the rating scale if the patient did not perform the identified self care task independently. This allowed documentation of any change in the carer's "effort" needed for a particular activity.

STATISTICAL ANALYSIS

Descriptive data is given for changes in the functional assessments, the scoring of passive range of finger movement, and change in pain before and after treatment. Student's $t$ test was used to detect significant change in the range of joint movement. Wilcoxon non-parametric analysis was used to analyse changes in spasticity as graded by the modified Ashworth scale.

\section{Results}

IMPAIRMENTS

Before treatment, 16 of 17 patients had a modified Ashworth rating of biceps spasticity of at least 4. All patients had a modified Ashworth grading of 5 for spasticity of the forearm finger flexors. There was a significant improvement in the grading of biceps spasticity after treatment (pretreatment median score 5, range 2-5; posttreatment median score 3 , range $2-4 ; \mathrm{P}=$ 0.001 ). There was also a significant improvement in the grading of forearm finger flexor spasticity after treatment (pretreatment median score 5, range all 5; post-treatment median score 4 , range $2-5 ; P=0.005$ ). It is notable that four out of seven patients reported improvement in self care despite no change in the Ashworth grading of forearm finger flexor spasticity.

The mean range of passive shoulder abductionor adduction was $65^{\circ}$ before treatment. The mean improvement after treatment was $17^{\circ}$ (95\% confidence interval (95\% CI) $7 \cdot 2$ $26.4 ; P=0.002$ ), with four of the 17 patients having at least a $30^{\circ}$ improvement in this range. The mean range of passive shoulder flexion and extension was $87^{\circ}$ before treatment. The mean improvement after treatment was $17^{\circ}(95 \% \mathrm{CI}$ $-3-33 ; P=0.09$ ) with five out of 17 patients having at least a $30^{\circ}$ improvement in this range. The mean range of passive elbow flexion and extension was $113^{\circ}$ before treatment. The mean improvement after treatment was $16^{\circ}$ (95\% CI 7.2-28.8; $\mathrm{P}=0.04)$ with three out of 17 patients having at least a $30^{\circ}$ improvement in this range. Before treatment, nine out of 17 patients had a clinically fixed flexion deformity at the elbow (mean flexion deformity, $43^{\circ}$; range of flexion deformity $10^{\circ}-80^{\circ}$ ). After treatment, the elbow could be fully passively extended in four out of these nine patients. The mean range of passive wrist dorsiflexion and palmar flexion was $60^{\circ}$ before treatment. The mean improvement after treatment was $31^{\circ}(95 \%$ CI $20 \cdot 1-$ 43.4; $\mathrm{P}<0.001$ ) with nine out of 17 having at least a $30^{\circ}$ improvement in this range after treatment. In six out of 17 patients the wrist could not be passively dorsiflexed beyond neutral before treatment. After treatment in four out of these six patients passive dorsiflexion beyond neutral was possible.

Before treatment, 14 out of 17 patients had flexed fingers at rest preventing easy insertion of one finger into the palm (fig 1) with none

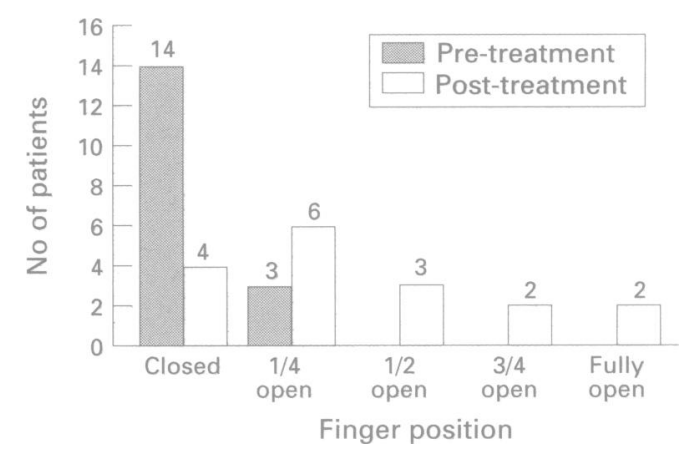

Figure 1 Finger position of hemiplegic hand at rest, before, and after treatment. 
Table 2 The effect of botulinum toxin treatment on the functional difficulties reported by patients or their carers

\begin{tabular}{|c|c|c|c|c|}
\hline Type of activity & $\begin{array}{l}\text { No of patients } \\
\text { reporting } \\
\text { difficulty with } \\
\text { the activity }\end{array}$ & $\begin{array}{l}\text { No of patients } \\
\text { dependent on } \\
\text { carer for the } \\
\text { activity }\end{array}$ & $\begin{array}{l}\text { No with } \\
\text { improvement } \\
\geqslant 2 \text { grades } \\
\text { of difficulty }\end{array}$ & $\begin{array}{l}\text { No of patients } \\
\text { becoming } \\
\text { independent } \\
\text { of their carer } \\
\text { for the activity }\end{array}$ \\
\hline $\begin{array}{l}\text { Cleaning palm of } \\
\text { affected hand }\end{array}$ & $17 / 17$ & $13 / 17$ & $13 / 17$ & $5 / 13$ \\
\hline $\begin{array}{l}\text { Cutting finger nails of } \\
\text { affected hand }\end{array}$ & $17 / 17$ & $13 / 17$ & $10 / 17$ & $2 / 13$ \\
\hline $\begin{array}{l}\text { Putting affected arm through } \\
\text { the sleeve of shirt or coat }\end{array}$ & $16 / 17$ & $11 / 16$ & $4 / 16$ & $2 / 11$ \\
\hline Standing balance & $4 / 17$ & - & $1 / 4$ & - \\
\hline Walking balance & $3 / 17$ & - & $0 / 3$ & - \\
\hline $\begin{array}{l}\text { Cleaning the armpit of } \\
\text { affected arm } \\
\text { Putting glove on }\end{array}$ & $2 / 17$ & $2 / 2$ & $1 / 2$ & $0 / 2$ \\
\hline affected hand & $1 / 17$ & $0 / 1$ & $0 / 1$ & - \\
\hline Turning in bed & $1 / 17$ & $0 / 1$ & $0 / 1$ & - \\
\hline
\end{tabular}

having sufficient range of passive extension of the fingers to allow easy placement of four fingers on the palm. After treatment not only could at least one finger be easily inserted into the palm in 10 out of these 14 patients but also in 10 of the 17 patients full passive extension of the fingers was possible allowing easy placement of four fingers on the palm.

Shoulder pain on passive movement was reported by nine out of $17(53 \%)$ patients before treatment. After treatment six out of these nine patients reported improvement, with complete resolution of shoulder pain in two patients. Elbow pain on passive movement was reported by three out of 17 patients before treatment. After treatment, all reported improvement in elbow pain. Wrist pain on passive movement was reported by six out of 17 patients before treatment. After treatment, this improved in five out of the six patients.

\section{DISABILITIES}

Table 2 shows the activities that patients reported difficulty with before treatment. This
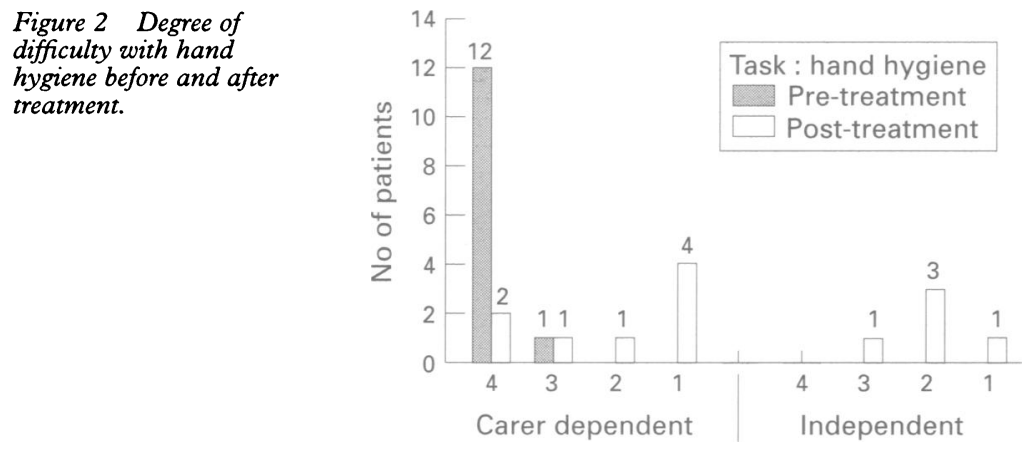

Figure 3 Degree of difficulty putting hemiplegic arm through sleeves before and after treatment. table also shows the number of patients that were dependent on their carers for each particular activity. All 17 patients had reported difficulty with cleaning the palm and cutting the finger nails of the affected hand; 13 were dependent on their carer to carry out these activities. Sixteen of 17 had difficulty with putting the arm through the sleeve of a shirt or coat, and one in 17 had difficulty putting on gloves. Three patients who could walk thought that spasticity in their arm caused elbow flexion while walking and this affected balance.

Overall, 14 of 17 patients reported some functional benefit after treatment with botulinum toxin. Table 2 shows the number of patients who improved two or more levels of difficulty after treatment. To illustrate reduction in dependence on the carer for the activities described, figs 2 and 3 show the changes in difficulty with hand hygiene and putting the arm through sleeves in patients who were dependent on their carers for these activities before treatment. Five out of 13 patients who were initially dependent on their carer to carry out hand hygiene became independent. Similarly two out of 11 patients who depended on the carer to put their affected arm through the sleeve of a shirt or coat became independent.

\section{FOLLOW UP}

In the 14 patients who reported functional benefit, an effect was apparent two to three days after treatment. Three patients who reported benefit at two weeks after treatment failed to attend for continued follow up. In the remaining 11 patients, functional improvement lasted between four weeks and 47 weeks. Sensation in both arms was clinically assessed before and after treatment and no change was noted. No adverse effects, such as flu-like symptoms and swallowing difficulties, were reported by any of the patients

\section{Discussion}

To fully assess the impact of local treatment for spasticity not only do changes in impairment have to be measured (for example, change in range of joint movement, clinical grading of spasticity, and pain), but also the effects of treatment on disability. A preliminary study of the use of botulinum toxin in stroke patients with upper limb spasticity by Hesse et $a l^{13}$ showed that certain self care activities such as hand hygiene did improve; however, little measurable effect was recorded on standardised functional assessments. We think that this disappointing conclusion arose through the use of global assessments of motor impairment and disability (Rivermead motor score $^{14}$ and Barthel index ${ }^{12}$ ) which are relatively insensitive to the type of improvement reported by patients. Moreover, the secondary effects of spasticity such as shoulder, elbow, or wrist joint pain (with consequent sleep disturbance and depression) may be poorly reflected in the impairment or disability scores used. Although local treatment may be effective, it is 
often difficult to quantify improvement if global assessments of function such as the Barthel index are used.

Patient defined goal assessments provide an alternative to the standard global assessments to quantify change in specific functional ability. The scale used in this study is based on the MACTAR ${ }^{15}$ which has been used to assess disability in rheumatoid arthritis. The results show that this scale can detect change in the degree of difficulty experienced by patients or carers in specific functional tasks. Although formal validation of this instrument is needed, the changes in difficulty reported by patients or carers nevertheless suggest that botulinum toxin may have a role in reducing specific upper limb disabilities. In addition, attainment of independence in certain aspects of self care as defined by the patient (for example, hand hygiene, putting affected arm through sleeves) can be achieved and are detected using this instrument.

Periarticular pain was also improved in some patients after treatment. Relief of severe spasticity of the biceps and forearm finger flexor muscles, and increased mobility of the joints are likely to contribute to pain relief after treatment. During this study, we also found that determining the amount of fixed shortening (contracture) in spastic muscles can be difficult as shown by improvement in range of passive elbow movement in patients who were previously considered to have a "fixed" flexion deformity.

As well as the local beneficial effects mentioned, improvement also occurred distant to the site of injection, as shown by the improvement in range of shoulder movement and relief of shoulder pain. Possible explanations for this improvement include: (1) local diffusion of toxin across fascial planes as occurs after its use in torticollis ${ }^{16}$ and (2) systemic effects. In vitro studies have shown retrograde axonal flow of botulinum toxin ${ }^{17}$ along the nerve fibre suggesting that spinal mechanisms may also be involved in the distant effect. ${ }^{18}$

There is risk of unwanted muscle weakness after botulinum toxin treatment. This is mainly related to local diffusion of toxin across muscle fascial boundaries although systemic toxin spread should not be excluded. Significant clinical adverse effects (dysphagia) can occur when botulinum toxin is used for the treatment of torticollis. This effect mainly relates to the close proximity of the treated muscles to those involved in swallowing. No such problems were reported in the patients treated in this study, indicating that total doses up to $1000 \mathrm{MU}$ of Dysport and $200 \mathrm{MU}$ of BOTOX $^{\mathbb{B}}$ may be safely given for upper limb spasticity.

Previous studies with botulinum toxin in torticollis suggest that the treatment effect usually lasts between three and six months. In this preliminary study some patients had functional benefit after treatment that apparently outlasted the "drug effect". Patient 9 (table 1 ) had continued functional benefit which lasted 47 weeks. We think that the prolonged treatment effect was related to a vigorous self exer- cise programme instituted by the patient herself. It was sometimes difficult to control for self initiated exercise programmes, as the patients who noticed improvement tended to carry out arm mobilisation exercises. This suggests that botulinum toxin may offer a window of opportunity to allow a passive mobilisation exercise programme to be instituted to maintain functional gains made after treatment. Clearly we need to explore this phenomenon further to capitalise on functional benefit gained after this treatment. It might be expected that the greatest impact would occur if botulinum toxin treatment was given earlier after stroke rather than later. Four out of 17 patients were treated within one year of stroke onset. One patient reported no reduction in disability. The other three patients had functional benefit lasting between four and 16 weeks. Three out of 17 patients were treated more than 10 years after their stroke and all showed benefit lasting between six and 10 weeks. Although from these limited data it is not possible to conclude that early treatment is likely to be more efficacious, we suspect that botulinum toxin has a role in the rehabilitation of the spastic upper limb during the early stages of rehabilitation as an adjunct to physiotherapy and occupational therapy, particularly when spasticity hinders arm function in patients with active upper limb movement.

This preliminary open study suggests that botulinum toxin is a safe and effective treatment for spasticity in this group of patients. Upper limb disability and pain can be reduced. Distant beneficial effects are also found. Validation of the patient oriented goal assessment is being carried out so that more robust statistical analysis can be applied. A randomised placebo controlled study is planned. There is clearly a need to measure spasticity more accurately and relate it to difficulty with specific activities including self care. Also, activities that cause increases in upper limb spasticity, such as walking and wheelchair propulsion, need to be explored further.

1 Gowland C. Management of hemiplegic upper limb. In: Brandstater ME, Basmajian JV, eds. Stroke rehabilitation. Baltimore: Williams and Wilkins, 1987:217-45.

2 Skeil DA, Barnes MP. The local treatment of spasticity. Clin Rehabil 1994;8:240-6.

3 Scott AB. Botulinum toxin injection of eye muscles to correct strabismus. Trans Am Ophthalmol Soc 1981;79: 734-70.

4 Scott AB, Kennedy RA, Stubbs HA. Botulinum toxin injection as a treatment for blepharospasm. Arch Ophthalmol 1985;103:347-50.

5 Flanders $M$, Chin D, Boghen D. Botulinum toxin: preferred treatment for hemifacial spasm. Eur Neurol 1993; 33;316-9.

6 Anderson TJ, Rivest J, Stell R, et al. Botulinum toxin treatment of spasmodic torticollis. $\mathcal{F} R$ Soc Med 1992;85: 524-9.

7 Jankovics J. Botulinum toxin in movement disorders. Current Opinions in Neurology 1994;7:358-66.

8 Das TK, Park DM. Effect of treatment with botulinum toxin on spasticity. Postgrad Med $\mathcal{F} 1989 ; 65: 208-10$.

9 Snow BJ, Tsui JK, Bhatt MH, Varelas M, Hashimoto SA, Calne DB. Treatment of spasticity with botulinum toxin: Calne DB. Treatment of spasticity with botulinum

10 Borodic GE. Botulinum toxin potency: a mystery resolved by the median paralysis unit. $\exists R$ Coll Phys 1994;87: by the

11 Bohannon RW, Smith MB: Interrater reliability of a modified Ashworth scale of muscle spasticity. Phys Ther 1987; fied Ashwort

12 Mahoney FI, Barthel DW. Functional evaluation: the Barthel index. MD Med f 1965;14:61-5. 
13 Hesse S, Friedrich H, Domasch C, Mauritz K. Botulinum toxin therapy for upper limb flexor spasticity: preliminary toxin therapy for upper limb flexor spas

14 Lincoln N, Leadbitter D. Assessment of motor function in stroke patients. Physiotherapy 1979;65:48-51.

15 Tuowell P, Bombardier C, Buchanan WW, Goldsmith CH, Grace E, Hanna B. The MACTAR patient preference disability questionnaire-an individualised functional priority approach for assessing improvement in physical disability in clinical trials in rheumatoid arthritis. f Rheumatol 1987;14:446-51.
16 Borodic GE, Joseph M, Fay L, Cozzolino D, Ferrante R. Botulinum toxin A for the treatment of spasmodic torticollis: dysphagia and regional toxin spread. Head Neck

Weigand H, Erdmann G, Wellhöner HH. ${ }^{125}$ I-labelled botulinum A neurotoxin: pharmocokinetics in cats, after linum A neurotoxin: pharmocokinetics in cats, after intramuscular injection.

18 Garner CG, Strube A, Witt TN, Gasser T, Oetal WH Time course of distant effects of local injections of botulinum toxin. Mov disord 1993;8:33-7.

\section{NEUROLOGY IN LITERATURE}

\section{Sciatica}

I suppose sciatica can be regarded as part of the neurologist's remit. Bennett is probably describing an S1 root syndrome. Many treatment options have been advanced for back pain. That used on Grigory appears remarkably successful-perhaps the fact that the secret medicine was laced with vodka had something to do with it!

Laurence Sterne, 1759, The life and opinions of Tristram Shandy gentleman

And how goes it with Thy Concubine-thy wife-and thy little ones o' both sides? And when did you hear from the old gentleman and Lady-your sister, aunt, uncle and cousins-I hope they have got better of their colds, coughs, claps, tooth-aches, fevers, stranguries, sciaticas, swellings, and sore eyes.

Fydodor Dostoyevsky, 1880, The brothers Karamazov And she treats Grigory with this secret medicine of hers three times a year, Sir, every time his lumbago gets so bad that he can't move, just as though he was paralyzed, Sir. Yes, Sir, three times a year. When this happens, she takes a towel, dips it in the infusion and rubs his back with it for half an hour, till it's bone dry and goes quite red and swollen. Then what's left over in the bottle she gives him to drink with a special prayer, Sir. Not all of it, though, for she leaves a drop or two over for herself, seeing as how it's such a rare occasion, Sir, and she drinks it, too. And both being strictly teetotal, Sir, they just drops off and they sleeps very soundly for a very long time. When Grigory wakes up, he's almost always well after it, but when Marfa wakes up she always has a headache from it, $\mathrm{Sir}$...

But quite unexpectedly Grigory woke up in the night, lay awake thinking for a moment and, though he immediately felt a sharp pain in the small of his back, sat up in bed.

Edith Wharton, 1911, Ethan Frome

"Mr Hale? Why, yes, you'll find him down home now. He ain't going to his work this forenoon. He woke up with a touch o' lumbago, and I just made him put on one of old Dr Kidder's plasters and set right up into the fire."

\section{Arnold Bennet, 1911, Hilda Lessways}

She had three genuine complaints, rheumatism, sciatica, and neuritis; they were all painful. The latest and worst was the neuritis, which had attacked her in the wrist, producing swollen joints that had to be fomented with hot water . .

On the previous day she had been sitting on the cold new oilcloth of the topmost stairs, minutely instructing a maid in the craft of polishing banisters. And the next morning an attack of acute sciatica had supervened. For a trifling indiscretion Sarah was thus condemned to extreme physical torture. Hilda had found her rigid on the bed. She suffered the severest pain in the small of the back and all down the left leg.

G D PERKIN Regional Neurosciences Centre, Charing Cross Hospital, Fulham Palace Road, 